Pacific Journal of Mathematic 


\title{
PIECEWISE CATENARIAN AND GOING BETWEEN RINGS
}

\author{
M. BrodmanN
}

The main purpose of this paper is to prove the following theorem. Let $R$ be a noetherian ring and $n$ a nonnegative integer. Then $R\left[X_{1}, \cdots, X_{n}\right]$ is a going-between ring (=GB) iff $R$ is $\mathbf{G B}$ and is $(n+1)$-piecewise catenarian.

In [7] Ratliff proved that all polynomial rings over an unitary commutative noetherian going-between-( $=\mathrm{GB})$-ring $R$ are again GB iff $R$ is catenarian (thus universally catenarian by [6, (3.8)] and [5, (2.6)]). (Recall that $R$ is called a GB-ring if for any integral extension $R^{\prime}$ of $R$ each adjacent pair of Spec $\left(R^{\prime}\right)$ retracts to an adjacent pair of $\operatorname{Spec}(R)$.)

In the meantime we showed that there are noetherian GB-rings which are not catenarian, thus giving a negative answer to a corresponding question of [7] (s. [2]). So it may be interesting to know more about the relations between the GB-property of polynomial rings and the chain structure of Spec $(R)$. In this note we shall investigate such a relation, thereby improving Ratliff's above result.

To formulate our statement, let us give the following

DeFinition $1 . \quad R$ is called n-piecewise catenarian $\left(=C_{n}\right)$. If $(R / P)_{Q}$ is catenarian for any pair $P, Q$ of $\operatorname{Spec}(R)$ related by a saturated chain $P=P_{0} \varsubsetneqq P_{1} \varsubsetneqq \cdots \varsubsetneqq P_{i}=Q$ of length $i \leqq n$.

Our main goal is to prove

THEOREM 2. Let $R$ be a noetherian ring and $n$ a nonnegative integer. Then $R\left[X_{1}, \cdots, X_{n}\right]$ is $\mathrm{GB}$ iff $R$ is $\mathrm{GB}$ and satisfies the property $C_{n+1}$.

Noticing that $R$ is catenarian iff it is $C_{n}$ for all $n>1$, this gives immediately the quoted result of Ratliff.

To prove 2 , let us introduce the following notations

3. (i ) $c(R)=$ set of lengths of maximal chains $P_{0} \varsubsetneqq P_{1} \varsubsetneqq \ldots$ of $\operatorname{Spec}(R)$ (s. [3], where $c(R)$ was investigated).

(ii) If $R$ is semilocal with Jacobson radical $J$, put $\hat{d}(R)=$ $\min \{\operatorname{dim}(\hat{R} / \hat{P})$, where $\hat{P}$ is a minimal prime of $\hat{R}\}, \hat{R}$ denoting the $J$-adic completion of $R$ (s. [1]). 
We also shall use the following characterization of GB-rings, whose proof is immediate from the basic results of [6] and [7].

Proposition 4. For a noetherian ring $R$ the following statements are equivalent:

(i) $R$ is GB.

(ii) For all $P, Q \in \operatorname{Spec}(R)$ with $P \subseteq Q$ the ring $T=(R / P)_{Q}$ is GB.

(iii) For all $T$ as in (ii) we have $c(T)=c(\hat{T})$.

(iv) For all $T$ as in (ii) we have $\min \mathrm{c}(\hat{T})=\hat{d}(T)=\min \mathrm{c}(T)$.

(v) For all $T$ as in (ii) which moreover are of dimension > one, we have $\hat{d}(R)>1$.

To prove 2 we start with the case $n=1$.

LEMma 5. Let $R$ be a noetherian ring. Then $R[X]$ is GB iff $R$ is GB and satisfies $C_{2}$. $(X)$.

Proof. " $\Longleftarrow$ " Let $R[X]$ be GB. Then so obviously is $R=R[X] /$

To show that $R$ satisfies $C_{2}$ let $P \varsubsetneqq Q \varsubsetneqq S$ be a saturated chain of $\operatorname{Spec}(R)$ such that $h t(S / P)>2$. We have to prove that $R[X]$ fails to be GB under this assumption. In replacing $R$ by $(R / P)_{S}$ we may restrict ourselves to show that $R[X]$ is not $\mathrm{GB}$, where $(R, M)$ is a local domain of dimension $>2=\min \mathrm{c}(R)$, which moreover is GB.

Let $\hat{P}$ be a minimal prime of $\hat{R}$ whose dimension is 2 (such a $\hat{P}$ exists by (4)). Choose $a \in M-(O)$ and let $b \in M$ be outside of all minimal prime divisors of $a R$ and of $a \hat{R}+\hat{P}$. Put $f=a X+b$. Then we first have the inclusion $f \hat{R}[X]+\hat{P} \hat{R}[X] \subseteq M \hat{R}[X]$ showing that there is a minimal prime $\widetilde{Q}$ of $f \hat{R}[X]+\hat{P} \hat{R}[X]$ with $\widetilde{Q} \cong M \hat{R}[X]$. As $h t(\widetilde{Q} / \hat{P} \hat{R}[X])=1$, we have the following two possibilities for $\hat{Q}=\widetilde{Q} \cap R$ :

$\widehat{Q}=\hat{P}$, or $h t(\hat{Q} / \hat{P})=1$ and $a$ and $b$ belong to $\hat{Q}$. By our choice of $a$ and $b$ we may exclude the second case. So, as $h t(\widetilde{Q} / \hat{P} \hat{R}[X])=$ $1, \widetilde{Q}$ is a minimal prime of $f \hat{R}[X]$. But now $h t(M \hat{R}[X] / \hat{P} \hat{R}[X])=$ $h t(M \hat{R} / \hat{P})$ implies that $h t(M \hat{R}[X] / \widetilde{Q})=1$. From this we conclude that $f\left(\hat{R}[X]_{M \hat{R}_{[X]}}\right)$ has a minimal prime divisor of dimension one. On the other hand we have a canonical isomorphism of $R[X]$-algebras

$$
\left(\hat{R}[X]_{M \hat{R}[X]}\right)^{\wedge} \simeq\left(R[X]_{M R[X]}\right)^{\wedge},
$$

which shows that $f\left(R[X]_{M R[X]}\right)^{\wedge}$ has a minimal prime divisor of dimension one.

Let us denote this prime divisor by $S^{\prime}$ and put $S=S^{\prime} \cap R[X]_{M R[X]}$. 
Then, by the flatness of completion, $S^{\prime}$ is a minimal prime divisor of $S R[X]_{M R[X]}$ and $S$ is a minimal prime divisor of $f R[X]_{M R[X]}$. Our choice of $a$ and $b$ implies that $S^{\prime \prime}=R[X] \cap(R-(O))^{-1} f R[X]$ is the unique minimal prime divisor of $f R[X]$. Thus $S=S^{\prime \prime} R[X]_{M R[X]}$ is the unique minimal prime divisor of $f R[X]_{M R[X]}$. This implies that $T=R[X]_{M R[X]} / S$ is of dimension $h t(M)-1>1$ but such that $\hat{d}(T) \leqq$ $\operatorname{dim}\left(S^{\prime}\right)=1$. So, by (i) $\Rightarrow(\mathrm{v})$ of $(4) R[X]$ is not GB.

“ $\Longrightarrow$ By 4 we may restrict ourselves to prove

6. Let $(R, M)$ be a noetherian local domain which is $\mathrm{GB}$ and $C_{2}$ and let $U$ be a simply generated extension domain of $R$. Let $N \in \operatorname{Spec}(U)$ such that $N \cap R=M$ and $h t(N)>1$. Then it holds $\hat{d}\left(U_{N}\right)>1$.

Put $U_{N}=T$. If $\hat{d}(R) \leqq 24$ shows that $\min \mathrm{c}(R) \leqq 2$. Thus the $C_{2}$ property of $R$ and 4 imply that $\hat{d}(R)=\operatorname{dim}(R)$, hence that $R$ is quasiunmixed. But then $T$ is also quasinmixed ([5], Cor. (2.6)] and therefore satisfies $\hat{d}(T)=h t(N)>1$.

If $\hat{d}(R)>2$ we use the inequality

$$
\hat{d}(T)-\hat{d}(R) \geqq \operatorname{deg} \operatorname{trans}(T: R)-\operatorname{deg} \operatorname{trans}(U / N: R / M)
$$

(s. [1, (4.4) (i)]), which gives the result as both of its right hand terms are 0 or 1 .

Next we give two results which deal with the $C_{n}$ property of polynomial rings.

LEMMA 7. Let $(R, M)$ be a noetherian local domain and let $(O)=P_{0} \varsubsetneqq P_{1} \varsubsetneqq \cdots \varsubsetneqq P_{n}=M(n \geqq 2)$ be a maximal chain of $\operatorname{Spec}(R)$ such that $h t\left(M / P_{n-2}\right)=2$. Then there is a saturated chain $Q_{0} \varsubsetneqq$ $Q_{1} \varsubsetneqq \cdots \varsubsetneqq Q_{n-2} \varsubsetneqq Q_{n-1}=M R[X]$ satisfying:

$Q_{i} \cap R=P_{i}$ and $h t\left(M R[X] / Q_{i}\right)=h t\left(M / P_{i}\right)-1$ for $i=1, \cdots, n-2$.

Proof. Choose $a \in M-P_{n-2}$ and let $b \in M$ be outside of all minimal prime divisors of $a R+P_{i}$ for $i=1, \cdots, n-2$. Put $f=$ $a X+b$. Then for all indices $i$ in question $f R[X]+P_{i} R[X]$ has exactly one minimal prime divisor, say $Q_{i}$. This implies that $Q_{0} \varsubsetneqq$ $Q_{1} \varsubsetneqq \cdots \varsubsetneqq Q_{n-2} \varsubsetneqq M R[X], Q_{i} \cap R=P_{i}$ and $h t\left(M R[X] / Q_{i}\right)=h t\left(M / P_{i}\right)-$ 1 for $i=1, \cdots, n-2$.

Thus it remains to prove that $h t\left(Q_{i} / Q_{i-1}\right) \leqq 1$ for $1 \leqq i \leqq n-2$. But this is immediately clear from $h t\left(Q_{i} / P_{i-1} R[X]\right) \leqq 2$, a relation due to $Q_{i} \cap R=P_{i}$ and the fact that $R$ is noetherian. 
Corollary 8. Let $R$ be a noetherian ring. Assume that for each maximal ideal $M$ of $R$ the ring $R[X]_{M R[X]}$ satisfies $C_{n-1}$, where $n$ is an integer $>2$. Then $R$ satisfies $C_{n}$.

Proof. Let $P, Q \in \operatorname{Spec}(R)$ be such that $P \subset Q$ and such that $2 \leqq \min \mathrm{c}\left(T=(R / P)_{Q}\right)=m \leqq n$. We have to show that $\operatorname{dim}(T)=m$. Obviously we may replace $R$ by $T$, hence assume that $(R, M)$ is a local domain with $\min \mathrm{c}(R)=m \leqq n$, and restrict ourselves to prove that $h t(M)=m$.

Thus let $(O)=P_{0} \varsubsetneqq \cdots \varsubsetneqq P_{m}=M$ be a maximal chain of $\operatorname{Spec}(R)$. Then it is clear that $P_{m-2} R[X] \varsubsetneqq P_{m-1} R[X] \varsubsetneqq M R[X]$ form a saturated chain of Spec $(R[X])$, hence, by the $C_{2}$ property of $R[X]$, that $h t\left(M R[X] / P_{m-2} R[X]\right)=2$. This shows that $h t\left(M / P_{m-2}\right)=2$, and so we may choose a chain $Q_{0} \varsubsetneqq Q_{1} \varsubsetneqq \cdots Q_{m-2} \varsubsetneqq Q_{m-1}=M R[X]$ as in 7. Now $h t\left(M R[X] / Q_{0}\right)=h t(M)-1$ and $h t\left(M R[X] / Q_{0}\right)=m-1$ (this latter is implied by the $C_{n-1}$ property of $\left.R[X]_{M R[X]}\right)$ prove the result.

Lemma 9. Let $R$ be a noetherian GB ring which satisfies $C_{n}$ for an integer $n \geqq 2$. Then $R[X]$ satisfies $C_{n-1}$.

Proof. As each ring is $C_{1}$, we may assume that $n>2$. Thus let $\widetilde{P}, \widetilde{Q} \leqq \operatorname{Spec}(R[X])$ such that $\widetilde{P} \subset \widetilde{Q}, 2 \leqq m=\min \mathrm{c}((R[X] / \widetilde{P}) \widetilde{Q}) \leqq$ $n-1$. Then we have, with $P=\widetilde{P} \cap R, Q=\widetilde{Q} \cap R$ :

$$
\operatorname{minc}\left((R[X] /(P))_{\tilde{Q}}\right) \leqq m+1 \text {, if } \widetilde{Q} \neq Q R[X],
$$

and

$$
\min \mathrm{c}\left((R[X] /(P))_{(Q, X)}\right) \leqq m+2 \text {, if } \widetilde{Q}=Q R[X] .
$$

Applying [3,(3.7)] we get $\hat{d}\left((R / P)_{Q}\right) \leqq m+1$. As $R$ is GB, (i) $\Rightarrow$ (iv) of 4 shows that $\min \mathrm{c}\left((R / P)_{Q}\right) \leqq m+1 \leqq n$, and the fact that $R$ is $C_{n}$ implies that $T=(R / P)_{Q}$ is catenarian. As $T$ is GB it therefore is even universally catenarian, and so finally $(R[X] / \widetilde{P})_{\widetilde{Q}}$ is catenarian.

REMARK 10. Noetherian $C_{n}$ rings appearently never have been studied for their own sake. $C_{n}$ seems to be related to GB in general, as the GB property of $R$ is easily proved to be a necessary hypothesis in (9) if $n>2$. Note also that in general the properties $C_{n}$ and $C_{n+1}$ are independent (s. [2]) even for quasiexcellent GB domains.

Now we may prove our final result, from which 2 follows cleraly. 
Proposition 11. Let $R$ be a noetherian ring and let $n \in N$. Then the following statements are equivalent:

(i) $R$ is GB and satisfies $C_{n}$.

(ii) $R\left[X_{1}, \cdots, X_{m}\right]$ is GB and $C_{n-m}$ for all $m<n$.

(iii) $R\left[X_{1}, \cdots, X_{n-1}\right]$ is GB.

Proof. "(i) $\Rightarrow$ (ii)" is immediately proved by induction on $m$, in making use of 5 and 9.

"(ii) $\Rightarrow$ (iii)" is clear.

“(iii) $\Rightarrow$ (i)" Use 5 and 8 to make induction on $n$.

To conclude this paper, let us note that the arguments in 5 give rise to an easy proof of the following result of Ratliff [7].

CoROLlaRY 12. Let $R$ be a noetherian ring. Then $R[X]$ is GB iff $R[X]_{M R[X]}$ is GB for all maximal ideals $M$ of $R$.

Proof. If $R[X]_{M R[X]}$ is GB for all $M$ in question, so is $R_{M}$, hence $R$. But to prove " $\Longleftarrow "$ of 5 we obviously only made use of the GB property of the rings $R[X]_{M R[X]}$. So we see that $R$ is $C_{2}$ and 5 gives the result.

\section{REFERENCES}

1. M. Brodmann, On some results of L. J, Ratliff, Comp. Math., 36, 1 (1973), 83-99.

2. - A particular class of regular domains, to appear in J. of Algebra.

3. S. McAdam, Saturated chains in noetherian rings, Indiana Univ. Math. J., 23 (1973/74), 719-728.

4. L. J. Ratliff Jr., On quasiunmixed semilocal rings and the altitude formula, Amer. J. Math., 87, 2(1965), 278-284.

5. — On quasiunmixed domains, the altitude formula and the chain condition for prime ideals (II), Amer. J. Math., 92 (1970), 99-144.

6. - Going between rings and contractions of saturated chains of prime ideals, Rocky Mountain J. Math., 7, 4 (1977), 777-787.

7. — $A(X)$ and GB-noetherian rings, to appear.

Received October 3, 1978. Author supported by Schweizerischer Nationalfonds zur Förderung der Wissenschaftlichen Forschung.

DER UNIVERSITÄT

ROXELER STRASSE 64

BRD-44 MuENSTER

W. Germany 



\title{
PACIFIC JOURNAL OF MATHEMATICS
}

\section{EDITORS}

\author{
DoNALD BABBITT (Managing Editor) \\ University of California \\ Los Angeles, CA 90024 \\ HUGo RossI \\ University of Utah \\ Salt Lake City, UT 84112 \\ C. C. MOORE and ANDREW OGG \\ University of California \\ Berkeley, CA 94720
}

J. DugundJI

Department of Mathematics

University of Southern California

Los Angeles, CA 90007

R. FINN and J. Milgram

Stanford University

Stanford, CA 94305

\section{ASSOCIATE EDITORS}
E. F. BeCKenbach
B. H. NeumanN
F. WOLF
K. YoshidA

\section{SUPPORTING INSTITUTIONS}

\author{
UNIVERSITY OF BRITISH COLUMBIA \\ CALIFORNIA INSTITUTE OF TECHNOLOGY \\ UNIVERSITY OF CALIFORNIA \\ MONTANA STATE UNIVERSITY \\ UNIVERSITY OF NEVADA, RENO \\ NEW MEXICO STATE UNIVERSITY \\ OREGON STATE UNIVERSITY \\ UNIVERSITY OF OREGON
}

\author{
UNIVERSITY OF SOUTHERN CALIFORNIA \\ STANFORD UNIVERSITY \\ UNIVERSITY OF HAWAII \\ UNIVERSITY OF TOKYO \\ UNIVERSITY OF UTAH \\ WASHINGTON STATE UNIVERSITY \\ UNIVERSITY OF WASHINGTON
}

The Supporting Institutions listed above contribute to the cost of publication of this Journal, but they are not owners or publishers and have no responsibility for its content or policies.

Mathematical papers intended for publication in the Pacific Journal of Mathematics should be in typed form or offset-reproduced, (not dittoed), double spaced with large margins. Please do not use built up fractions in the text of the manuscript. However, you may use them in the displayed equations. Underline Greek letters in red, German in green, and script in blue. The first paragraph or two must be capable of being used separately as a synopsis of the entire paper. Please propose a heading for the odd numbered pages of less than 35 characters. Manuscripts, in triplicate, may be sent to any one of the editors. Please classify according to the scheme of Math. Reviews, Index to Vol. 39. Supply name and address of author to whom proofs should be sent. All other communications should be addressed to the managing editor, or Elaine Barth, University of California, Los Angeles, California, 90024.

50 reprints to each author are provided free for each article, only if page charges have been substantially paid. Additional copies may be obtained at cost in multiples of 50 .

The Pacific Journal of Mathematics is issued monthly as of January 1966. Regular subscription rate: $\$ 84.00$ a year $(6$ Vols., 12 issues). Special rato: $\$ 42.00$ a year to individual members of supporting institutions.

Subscriptions, orders for numbers issued in the last three calendar years, and changes of address shoud be sent to Pacific Journal of Mathematics, P.O. Box 969, Carmel Valley, CA 93924, U.S.A Old back numbers obtainable from Kraus Periodicals Co., Route 100, Millwood, NY 10546.

\section{PUBLISHED BY PACIFIC JOURNAL OF MATHEMATICS, A NON-PROFIT CORPORATION}

Printed at Kokusai Bunken Insatsusha (International Academic Printing Co., Ltd.). 8-8, 3-chome, Takadanobaba, Shinjuku-ku, Tokyo 160, Japan. 


\section{Pacific Journal of Mathematics}

\section{Vol. 86, No. 2 December, 1980}

Graham Donald Allen, David Alan Legg and Joseph Dinneen Ward, Hermitian

liftings in Orlicz sequence spaces ............................... 379

George Bachman and Alan Sultan, On regular extensions of measures ........ 389

Bruce Alan Barnes, Representations Naimark-related to $*$-representations; a

correction: "When is a representation of a Banach $*$-algebra

Naimark-related to a $*$-representation?" ........................ 397

Earl Robert Berkson, One-parameter semigroups of isometries into $H^{p} \ldots \ldots .403$

M. Brodmann, Piecewise catenarian and going between rings ............ 415

Joe Peter Buhler, A note on tamely ramified polynomials ............... 421

William Lee Bynum, Normal structure coefficients for Banach spaces ........ 427

Lung O. Chung, Biharmonic and polyharmonic principal functions ......... 437

Vladimir Drobot and S. McDonald, Approximation properties of polynomials

with bounded integer coefficients .............................

Giora Dula and Elyahu Katz, Recursion formulas for the homology of

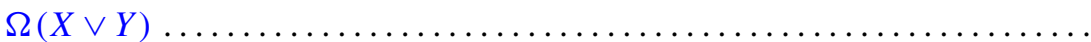

John A. Ernest, The computation of the generalized spectrum of certain Toeplitz operators ...................................... 463

Kenneth R. Goodearl and Thomas Benny Rushing, Direct limit groups and the

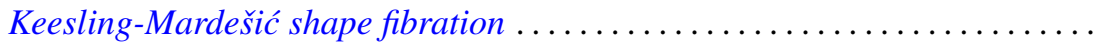

Raymond Heitmann and Stephen Joseph McAdam, Good chains with bad contractions

Patricia Jones and Steve Chong Hong Ligh, Finite hereditary near-ring-semigroups .

Yoshikazu Katayama, Isomorphisms of the Fourier algebras in crossed

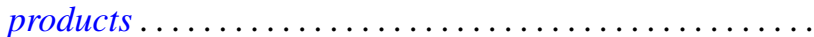

Meir Katchalski and Andrew Chiang-Fung Liu, Symmetric twins and common transversals. .

Mohammad Ahmad Khan, Chain conditions on subgroups of LCA groups ....

Helmut Kröger, Padé approximants on Banach space operator equations ...

Gabriel Michael Miller Obi, An algebraic extension of the Lax-Milgram theorem . .

S. G. Pandit, Differential systems with impulsive perturbation .

Richard Pell, Support point functions and the Loewner variation ...

J. Hyam Rubinstein, Dehn's lemma and handle decompositions of some

4-manifolds

James Eugene Shirey, On the theorem of Helley concerning finite-dimensional subspaces of a dual space.

Oved Shisha, Tchebycheff systems and best partial bases.... 